\title{
High gradient experiments with $X$-band cryogenic copper accelerating cavities
}

\author{
A. D. Cahill* and J. B. Rosenzweig \\ UCLA Los Angeles, California 90095, USA \\ V. A. Dolgashev, S. G. Tantawi, and S. Weathersby \\ SLAC Menlo Park, California 94025, USA
}

(Received 14 December 2017; published 23 October 2018)

\begin{abstract}
Vacuum radio-frequency (rf) breakdown is one of the major factors that limit operating accelerating gradients in rf particle accelerators. The occurrence of rf breakdowns was shown to be probabilistic, and can be characterized by a breakdown rate. Experiments with hard copper cavities showed that harder materials can reach larger accelerating gradients for the same breakdown rate. We study the effect of cavity material on rf breakdowns with short $X$-band standing wave accelerating structures. Here we report results from tests of a structure at cryogenic temperatures. At gradients greater than $150 \mathrm{MV} / \mathrm{m}$ we observed a degradation in the intrinsic cavity quality factor, $Q_{0}$. This decrease in $Q_{0}$ is consistent with $\mathrm{rf}$ power being absorbed by field emission currents, and is accounted for in the determination of accelerating gradients. The structure was conditioned up to an accelerating gradient of $250 \mathrm{MV} / \mathrm{m}$ at $45 \mathrm{~K}$ with $10^{8} \mathrm{rf}$ pulses and a breakdown rate of $2 \times 10^{-4} / \mathrm{pulse} / \mathrm{m}$. For this breakdown rate, the cryogenic structure has the largest reported accelerating gradient. This improved performance over room temperatures structures supports the hypothesis that breakdown rate can be reduced by immobilizing crystal defects and decreasing thermally induced stresses.
\end{abstract}

DOI: 10.1103/PhysRevAccelBeams.21.102002

\section{INTRODUCTION}

Accelerating gradient is one of the major parameters that determine the cost and viability of accelerator projects, such as large scale linear colliders for high energy physics and high brightness electron sources of free electron lasers (FELs), for example the Linac Coherent Light Source (LCLS) [1]. The major factor limiting larger accelerating gradients is vacuum rf breakdown [2-5], therefore breakdown physics is an active field of study with many contributors.

The accelerating gradient of the long-lived SLAC S-band linac is about $17 \mathrm{MV} / \mathrm{m}[6]$. During development of the Next Linear Collider (NLC)/Global Linear Collider (GLC), an $X$-band test accelerator operated at $65 \mathrm{MV} / \mathrm{m}$ unloaded gradient $[4,7]$. The CERN based Compact Linear Collider (CLIC) design requires $100 \mathrm{MV} / \mathrm{m}$ loaded gradient at $12 \mathrm{GHz}$ in accelerating structures with heavy wakefield damping [8]. Advances in understanding limitations on

\footnotetext{
*acahill@physics.ucla.edu
}

Published by the American Physical Society under the terms of the Creative Commons Attribution 4.0 International license. Further distribution of this work must maintain attribution to the author(s) and the published article's title, journal citation, and DOI. accelerating gradient go beyond linear colliders. rf accelerators are used in applications such as inverse Compton scattering gamma ray sources [9], compact free-electron lasers (FELs) [10,11], compact medical linacs for hadron therapy [12], photo-rf guns [13], 4-th harmonic linearizers for FELs [14], rf deflectors [15-17], and rf undulators [18,19], all may benefit from larger accelerating gradients. For example, if an S-band rf photoinjector that operates with sustained rf surface electric fields of $250 \mathrm{MV} / \mathrm{m}$ on the cathode is used as the electron source for LCLS [1], the required undulator length could be reduced by over $50 \%$ [20].

Early work by Loew et al. [21] and Balakin et al. [22] considered the rf breakdowns to be directly linked to peak electric field. Later, during the NLC/GLC work the statistical nature of $\mathrm{rf}$ breakdown became apparent $[4,7,23,24]$. For most accelerating structures at comparable rf power and pulse shape, the number of $\mathrm{rf}$ breakdowns per pulse is nearly steady or slowly decreasing over $10^{5}-10^{7}$ pulses. The breakdown rate (BDR) became one of the main quantitative requirements characterizing high gradient performance of linacs. For example: the CLIC linear collider requires $\mathrm{rf}$ breakdown probability to be less than $4 \times 10^{-7} / \mathrm{pulse} / \mathrm{m}$ for a loaded accelerating gradient of $100 \mathrm{MV} / \mathrm{m}$.

As technology progressed, sophisticated manufacturing and surface preparation techniques and systematic rf processing methods were developed [25-27]. As a result of this 
R\&D, practical 11.4 GHz Traveling Wave (TW) accelerating structures, which are CLIC prototypes, run at breakdown rates of about $10^{-6} / \mathrm{pulse} / \mathrm{m}$ at unloaded gradients up to $120 \mathrm{MV} / \mathrm{m}$ and $200 \mathrm{~ns}$ pulse length [28]. TW structures that include wakefield damping work at about $100 \mathrm{MV} / \mathrm{m}$ for similar breakdown rates $[28,29]$. Small copper standing wave (SW) structures at $11.4 \mathrm{GHz}$ have reached $175 \mathrm{MV} / \mathrm{m}$ accelerating gradients with breakdown rates of $10^{-3} / \mathrm{pulse} / \mathrm{m}$.

\section{A. Properties of rf breakdowns}

Presently, $X$-band structures are the most studied in terms of rf breakdowns [5,24,30-32]. We know that breakdown statistics depend on pulsed surface heating [33] and a numerous list of other factors, such as the peak electric field, the peak magnetic field [34], the peak Poynting vector [35], and hardness of the cavity material [36].

Typically, rf breakdowns can be separated into two categories: trigger and secondary breakdowns [37]. The secondary breakdowns occur in a chain after and appear to be caused by the trigger breakdowns. A secondary breakdown will occur within a short period of time after the trigger breakdown and can occur at significantly lower field levels. Commonly, the trigger breakdown rate is understood to be dependent on material properties and structure geometry, and secondary breakdowns are associated with damage caused by the trigger breakdowns. A publication analyzing the statistics of rf breakdowns in CLIC TW structures, showed the distribution of breakdowns in time can be analyzed by a double Poisson distribution and that pairs of breakdowns that are close temporally are also close spatially [38].

A review of existing experimental data with the goal of determining parameters that scale with the BDR of accelerating structures is reported in [35]. First, the BDR was empirically found to be dependent on accelerating gradient, $G$, as BDR $\propto G^{30}$ for many TW structures. Second, the $\mathrm{BDR}$ increases for longer pulse lengths, $t_{p}$, with $\mathrm{BDR} \propto t_{p}^{5}$ for the same accelerating gradient. It was found that the modified Poynting vector is correlated with the BDR [35]. A recent review of the conditioning process of several TW structures showed that they conditioned at the same rate and to the same gradients with the same number of rf pulses, but not with the number of accumulated breakdowns [39].

A study of SW structures with varying geometries showed that breakdown rates correlate with peak rf pulsed heating rather than with peak rf electric fields [40]. This motivated work on the physics of periodic rf pulsed heating. At SLAC, a cavity was designed such that sample disks were not exposed to surface electric fields, but had enhanced surface magnetic fields [41]. The results were that harder materials, such as non-annealed $\mathrm{CuAg}, \mathrm{CuCr}$, and $\mathrm{CuZr}$, exhibited significantly less damage than annealed copper samples, and also damage was apparent when the temperature of peak pulse heating reached greater than $50 \mathrm{~K}$. Subsequently, short SW structures constructed from hard $\mathrm{CuAg}$ achieved accelerating gradients of $200 \mathrm{MV} / \mathrm{m}$ with breakdown rates near $10^{-3} / \mathrm{pulse} / \mathrm{m}$ [36].

In another study, reported in [42], copper samples were prepared with the same heat treatment process as for accelerating structures, and then mechanically stressed for the same number of pulses as used for conditioning of accelerating structures. Comparison of the copper samples and autopsy of accelerating structures showed similar damage and mechanical hardening on the surface.

The current consensus is that vacuum rf breakdowns are caused by movements of crystal defects induced by periodic mechanical stress. The stress may be caused by pulsed surface heating and large electric fields. There are theoretical models [43,44] that predict the empirical BDR $\propto G^{30}$ power law dependence for room temperature copper structures. The same theory predicts that for lower temperatures the power law $\mathrm{BDR} \propto G^{\zeta}$ has an exponent $\zeta$ greater than 30, and thus stronger gradient dependence than at room temperature.

Therefore, from the current understanding of rf breakdown physics, decreasing crystal mobility and reducing thermally induced stress should lower breakdown rates. This can be accomplished by operating accelerating structures at cryogenic temperatures.

\section{B. Cryogenic accelerators}

One possible method to increase sustained electric fields in copper cavities is to cool them to temperatures below $77 \mathrm{~K}$, where the rf surface resistance and coefficient of thermal expansion decrease, while the yield strength (which correlates with hardness) and thermal conductivity increase $[45,46]$, all of which can affect the limits of sustained surface fields. The changes in rf surface resistance, coefficient of thermal expansion, and thermal conductivity decrease the pulsed surface heating and mechanical stress applied to the cavity material, the peak rf pulsed heating will decrease by over a factor of three by decreasing the temperature from room temperature to $45 \mathrm{~K}$ [47], and mechanical stress is proportional to the coefficient of thermal expansion times the peak pulsed heating. Increasing the yield strength decreases crystal mobility, while the changes in the other material properties decrease thermal stresses.

Of these properties, the decrease in rf surface resistance has been studied the most, which in cryogenic copper is well described by the theory of anomalous skin effect [48]. Measurements of the rf losses are in agreement with this theory to within $10 \%$ at a range of frequencies: 1.17, 1.4, 3 , 5.7, 9.3, 11.4, and $35 \mathrm{GHz}$ [49-57]. These experiments used the intrinsic quality factor, $Q_{0}$, of copper resonant cavities at very low fields and sub Watt rf power to measure the rf surface resistance.

However, there is little data on the sustainable electric fields of copper cavities at temperatures below $100 \mathrm{~K}$ with high input rf power, corresponding to surface electric fields greater than $75 \mathrm{MV} / \mathrm{m}$. The results of an experiment at 
$9.3 \mathrm{GHz}, 77 \mathrm{~K}$, with $150-300 \mathrm{~kW}$ input peak power found surface electric fields up to $50 \mathrm{MV} / \mathrm{m}$ [55]. In another study conducted at $5.7 \mathrm{GHz}, 20 \mathrm{~K}$, showed surface fields up to $65 \mathrm{MV} / \mathrm{m}$ [51]. However, an experiment at $3 \mathrm{GHz}, 77 \mathrm{~K}$ with surface electric fields up to $300 \mathrm{MV} / \mathrm{m}$ showed a decrease of $Q_{0}$ with increased fields [57]. The authors report that the decrease in $Q_{0}$ is correlated with surface magnetic fields and is possibly caused by multipactor discharge [58].

In the experiments above, the breakdown rate was not considered. A study conducted at CERN attempted to show the relation between rf breakdown rate and decreased temperatures. Cavities with frequencies between 21 and $39 \mathrm{GHz}$ showed no dependence of breakdown rates on temperature between 100 and $800 \mathrm{~K}$ [59].

In our experiments we found that lowering the temperature of copper accelerating cavities allows them to sustain larger rf surface electric fields with decreased probability for vacuum rf breakdowns $[60,61]$. We also observed that the $Q_{0}$ decreases when accelerating gradients increase above $150 \mathrm{MV} / \mathrm{m}$. We found that the explanation for the $Q_{0}$ degradation is dark current beam loading [62]. In this paper we present experiments conducted with a cryogenic accelerating structure, and the measured rf breakdown rates.

\section{CAVITY DESIGN}

Our cryogenic accelerating cavity is designed to have high fields in a single, middle cell. Fifty similar structures have been tested at SLAC [40,63-66]. The shape for the cryogenic cavity, 1C-SW-A2.75-T2.0-Cryo-Cu-SLAC, was based on the geometry with the smallest aperture, $a=2.75 \mathrm{~mm}$, which has the highest-shunt-impedance out of those tested at room temperature. The irises have an elliptical shape with thickness $2.0 \mathrm{~mm}$. We use the same geometry to directly compare our room temperature results with those presented

TABLE I. Parameters of periodic accelerating structure with $\pi$ phase advance per cell and dimensions of the Cryo-Cu-SLAC middle cell at $45 \mathrm{~K}$ and $293 \mathrm{~K}$. Fields are normalized to $E_{\text {acc }}=$ $250 \mathrm{MV} / \mathrm{m}$ for $f_{0}=11.424 \mathrm{GHz}$. Peak pulsed heating is calculated for a pulse with $150 \mathrm{~ns}$ flat gradient.

\begin{tabular}{lcc}
\hline \hline Parameter & $293 \mathrm{~K}$ & $45 \mathrm{~K}$ \\
\hline Q-Value & 8,590 & 29,000 \\
Shunt impedance $[\mathrm{M} \Omega / \mathrm{m}]$ & 102.891 & 347.39 \\
$H_{\max }[\mathrm{MA} / \mathrm{m}]$ & 0.736 & 0.736 \\
$E_{\max }[\mathrm{MV} / \mathrm{m}]$ & 507.8 & 507.8 \\
$E_{\text {acc }}[\mathrm{MV} / \mathrm{m}]$ & 250 & 250 \\
$H_{\max } Z_{0} / E_{\text {acc }}$ & 1.093 & 1.093 \\
Losses in a cell $[\mathrm{MW}]$ & 7.97 & 2.36 \\
Peak pulsed heating $(150 \mathrm{~ns})[\mathrm{K}]$ & 86.9 & 21.9 \\
$a[\mathrm{~mm}]$ & 2.75 & 2.75 \\
$a / \lambda$ & 0.105 & 0.105 \\
Iris thickness $[\mathrm{mm}]$ & 2 & 2 \\
Iris ellipticity & 1.385 & 1.385 \\
\hline \hline
\end{tabular}

TABLE II. Measured parameters of full Cryo-Cu-SLAC-\#2 shown in Fig. 1. Fields are normalized to $E_{\text {acc }}=250 \mathrm{MV} / \mathrm{m}$ in the middle cell at $45 \mathrm{~K}$.

\begin{tabular}{lc}
\hline \hline Parameter & Value \\
\hline$f_{0}[\mathrm{GHz}]$ & 11.4294 \\
Q-Value & 30,263 \\
$H_{\max }[\mathrm{MA} / \mathrm{m}]$ & 0.736 \\
$E_{\max }[\mathrm{MV} / \mathrm{m}]$ & 507.8 \\
$E_{\mathrm{acc}}[\mathrm{MV} / \mathrm{m}]$ & 250 \\
Coupling $\beta$ & 1.97 \\
Power lost in walls $[\mathrm{MW}]$ & 3.46 \\
\hline \hline
\end{tabular}

here. The structure is made of three cells with the highest fields in the middle cell, to localize rf breakdowns to that cell. The middle cell shape is designed to mimic the properties of a longer periodic accelerating structure.

The cryogenic cavity does not include a field probe, as they distort and amplify surface fields and degrade high power performance. To determine the electric fields we instead use the measured input rf power to the cavity (forward power), rf power reflected from the cavity, and signals from the current monitors which intercept the field emission currents.

rf power is coupled into the structure using a $\mathrm{TM}_{01}$ mode launcher [67]. For the design of the structure, we used properties of cryogenic copper measured in previous experiments [54]. The structure was designed to be critically coupled, $\beta=1$, at $96 \mathrm{~K}$ with $Q_{0}=19,100$. At $45 \mathrm{~K}$, the cryogenic cavity is over-coupled with $\beta=2.12$, and at $293 \mathrm{~K}$ undercoupled with $\beta=0.45$. The resonant frequency of the accelerating mode is $11.424 \mathrm{GHz}$ at $150 \mathrm{~K}$.

The rf parameters for a periodic structure with dimensions of the middle cell for $45 \mathrm{~K}$ and $293 \mathrm{~K}$ are in Table I, and the measured parameters for the tested cryogenic cavity at $45 \mathrm{~K}$ are in Table II. The electric and magnetic fields are shown in Fig. 1 and the surface fields are shown in Fig. 2. The peak pulsed heating is calculated using the following equation:

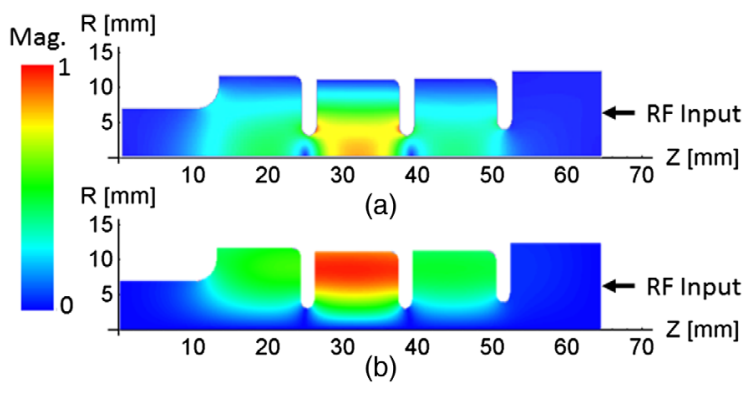

FIG. 1. Electric and magnetic fields for the cryogenic cavity. The fields are scaled to $3.46 \mathrm{MW}$ dissipated in the cavity walls. $\mathrm{T}=45 \mathrm{~K}$ and $Q_{0}=30,263$. (a) shows the surface electric fields, with a maximum $507 \mathrm{MV} / \mathrm{m}$. (b) shows the surface magnetic fields, with a maximum $736 \mathrm{kA} / \mathrm{m}$. 


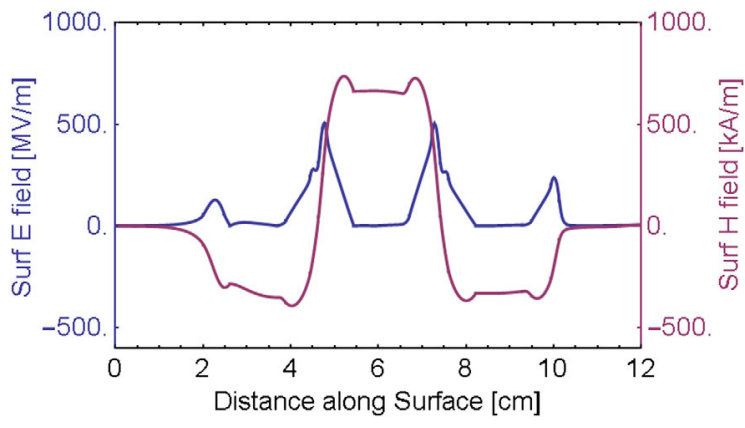

FIG. 2. Electric and magnetic fields along the surface for the $X$-band cryogenic cavity, scaled to $250 \mathrm{MV} / \mathrm{m}$ accelerating gradient in the middle cell.

$$
\Delta T=|H|^{2} \sqrt{t_{p}} \frac{R_{s}}{\sqrt{\pi \rho c_{\epsilon} k}},
$$

where $t_{p}$ is the rf pulse length for a square pulse, $R_{s}$ is the $\mathrm{rf}$ surface resistance, $\rho$ is the density, $c_{\epsilon}$ is the specific heat capacity, and $k$ is the heat conductivity. We assume these parameters do not change during the pulse.

\section{METHODS}

\section{A. Experimental setup}

The cavity was placed inside a vacuum cryostat and cooled by a pulse tube cryocooler, the Cryomech PT-415. The cold head of the cryocooler was in thermal contact with the cavity. The input waveguide is fed from the outside of the cryostat by a $\mathrm{TM}_{01}$ mode launcher [67]. A heat shield separates the SW structure from the rest of the cryostat. A diagram and photo of the cryostat are included in Figs. 3 and 5 .

We performed two experiments with identical structures, Cryo-Cu-SLAC-\#1 and Cryo-Cu-SLAC-\#2. The difference between the two was the following; in the first experiment the mode launcher was connected to the cavity with two rf chokes in a circular waveguide as described in [68]. These rf chokes create a gap in the circular waveguide that functions as a thermal break, preventing heat conduction from the room temperature waveguide to the cryogenic structure. However, the rf flanges and the current monitor shown in Fig. 3 connected the cryostat vacuum to that of the accelerating structure. This structure showed no improvement with decreased temperature and performed worse than previously tested structures. We speculated that gases from the vacuum of the cryostat contaminated the accelerating structure, causing and increase in the breakdown rate.

Following this experience, the feeding waveguide was modified to separate the cryostat vacuum from the accelerator vacuum. In the second experiment, we separate the cryostat and accelerator vacuum in the following ways, the rf chokes were removed and a thin 0.015 inch copper foil was introduced before the current monitor as shown in Fig. 3. The metal foil is thin enough to not significantly affect the near MeV field emitted electrons.

For low power measurements, we used a vector network analyzer (VNA), a Keysight N5242A. With the VNA we measured the dependence of the reflection coefficient on the rf frequency. Knowing this dependence we calculated $Q_{0}$ and other rf parameters using a linear equivalent circuit [69]. For high power tests, the rf source was a SLAC 50 MW XL-4 klystron, which was pulsed with a repetition rate of 5,10 , or $30 \mathrm{~Hz}$ and pulse length up to $500 \mathrm{~ns}$. The rf pulses are shaped in a specific way for this experiment, and we will use $t_{1}$ and $t_{2}$ as shown in Fig. 6 to describe the pulse, where the input rf pulse is the green curve in Fig. 6(a). The initial $150 \mathrm{~ns}$ is at high power, and then the power is dropped to a lower at time $t=t_{1}$ value that
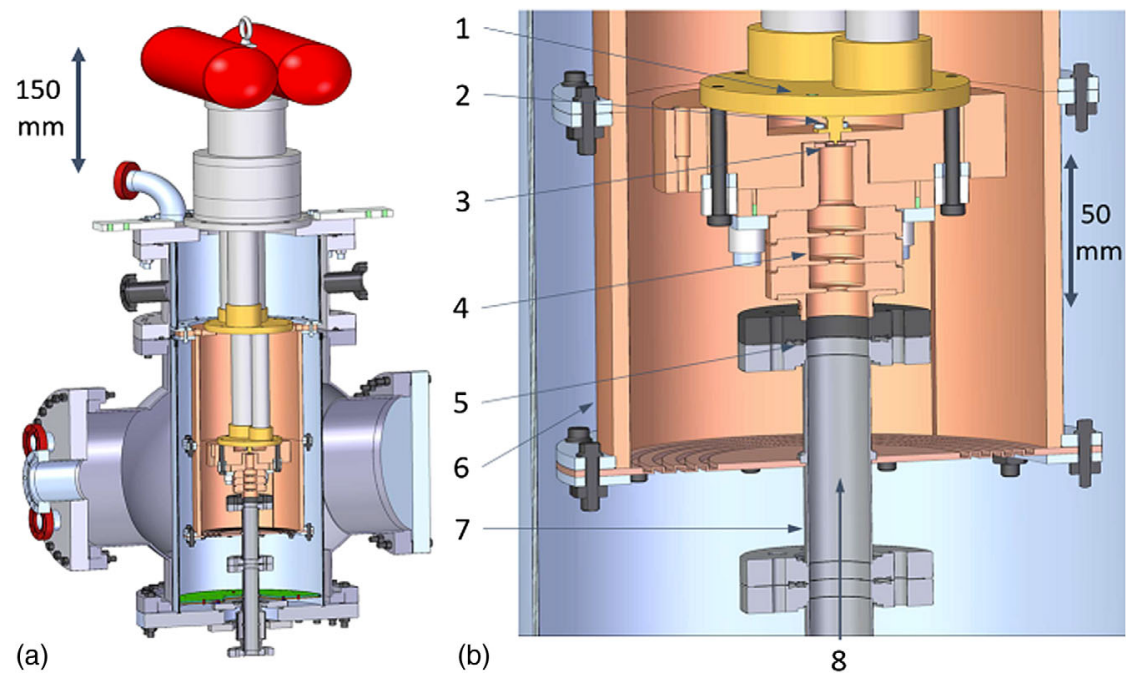

FIG. 3. (a) Solid model of the cryostat and (b) zoom in on Cryo-Cu-SLAC-\#2 in same model. (1) Cold head of cryocooler; (2) current monitor; (3) brazed metal foil; (4) Cryo-Cu-SLAC-\#2; (5) rf flange; (6) thermal shield; (7) Cu-plated stainless steel waveguide; (8) rf input. 


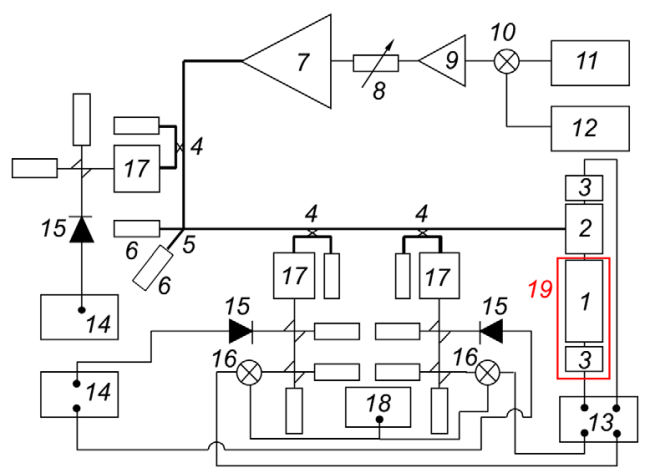

FIG. 4. Schematic diagram of the experimental setup: (1) CryoCu-SLAC-\#2; (2) $\mathrm{TM}_{01}$ mode launcher; (3) current monitors for field emission currents; (4) high power directional couplers; (5) waveguide $3 \mathrm{~dB}$ hybrid; (6) high power loads; (7) SLAC XL-5 X-band klystron; (8) variable attenuator; (9) booster traveling wave tube; (10) mixer for klystron signal shaping; (11) signal generator; (12) arbitrary function generator; (13) fast digitizer; (14) peak power meter; (15) probes of peak power meter; (16) mixer for data acquisition; (17) low pass filters; (18) signal generator for data acquisition; (19) cryostat envelope. Not numbered: cross guide directional couplers that couple signal between low pass filter (17) and probes (15) and (16) and low power rf loads which are connected to high power directional couplers (4) and cross guide directional couplers.

matches the steady-state power to maintain the accelerating gradient in the cavity. Therefore the accelerating gradient remains constant from $t_{1}$ to $t_{2}$. Figure 4 is a photo of the experimental setup.

Figure 5 shows a diagram of the high power circuit. The klystron (item-7 in Fig. 5) was connected to the cryostat by a network of WR90 waveguides, with a $3 \mathrm{~dB}$ hybrid (5) used to reduce large reflections to and from the klystron.

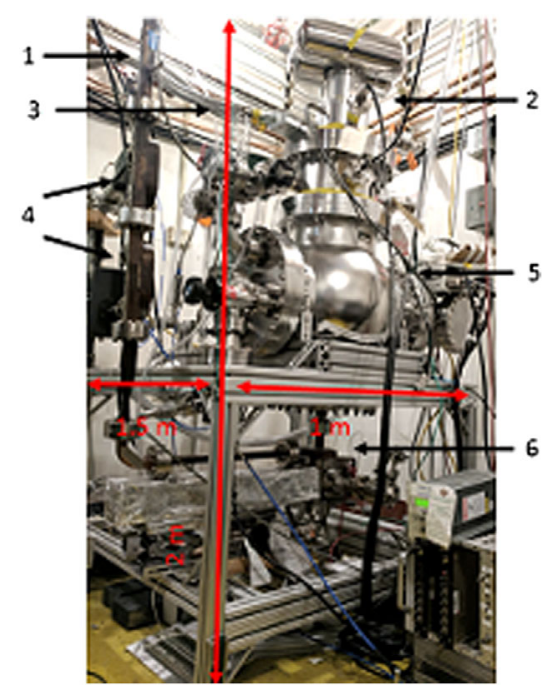

FIG. 5. Photo of experimental setup: (1) WR90 waveguide towards the klystron; (2) pulse tube cooler; (3) helium lines; (4) WR90 directional couplers; (5) vacuum chamber of the cryostat; (6) $\mathrm{TM}_{01}$ mode launcher.
The rf signals were sampled by two directional couplers (4) directly before the mode launcher. The forward and reflected signals were measured with a Keysight N1912A peak power meter (14) and downmixed to $115 \mathrm{MHz}$ (15) to be read by a fast digitizer (13). Two current monitors (3) on both sides of the cavity intercept the field emission electrons, and were connected via coaxial cables to the digitizer. The dark current measured will be a small portion of the dark current inside the cavity.

\section{B. Data processing and gradient reconstruction}

In our numerous previous experiments with room temperature cavities, the rf and dark current signals were well described by a linear equivalent circuit with a constant $Q_{0}$ [69], determined from VNA measurements. We will refer to this equivalent circuit as the linear model. However, during experiments with Cryo-Cu-SLAC-\#2, we found that $Q_{0}$ degrades during the rf pulse, which will the lower the peak accelerating gradient. We developed a model to account for the $Q_{0}$ degradation [62], which we will refer to as the nonlinear model. The decrease in $Q_{0}$ was found to be consistent with beam loading from the field emission currents.

To find the behavior of the electric field, we start with an eigensolution for electromagnetic fields in a perfectly conductive cavity with a resonant frequency of $\omega_{0}(t)$. Here, we assume that $\omega_{0}(t)$ is time-dependent. The magnitude of the electric field for the eigensolution is proportional to $\tilde{E}(t)$. Then there are two perturbations to the eigensolution, one the finite losses characterized by a time dependent quality factor $Q_{0}(t)$, and second one is a coupling to a waveguide with an external quality factor $Q_{E}$. We did not assume any specific model to describe the time-dependent losses or changing of the resonant frequency. Then we define a time dependent phasor of input power, $P_{\text {in }}(t)$, with constant frequency $\Omega$. Any time dependence of input frequency is contained in $P_{\text {in }}(t)$. For a detailed derivation of this equation, see [71].

When $Q_{0}$ and $\omega_{0}$ are time-dependent, $\tilde{E}(t)$, a variable proportional to the electric field inside the cavity, is governed by the following equation derived from the assumptions stated previously:

$$
\begin{aligned}
& \sqrt{\frac{8 P_{\text {in }}(t) \omega_{0}(t)^{3}}{\epsilon_{0} Q_{E}}}=\frac{d \tilde{E}(t)}{d t}\left[\frac{\omega_{0}(t)}{Q_{E}}+\Omega\left(\frac{1}{Q_{0}(t)}-2 i\right)\right] \\
& +\tilde{E}(t)\left\{\left[\omega_{0}(t)^{2}-\Omega^{2}\right]-i \Omega\left[\frac{\Omega}{Q_{0}(t)}+\frac{\omega_{0}(t)}{Q_{E}}\right]\right\}
\end{aligned}
$$

where $\Omega$ is the driving frequency of the input $\mathrm{rf}, P_{\text {in }}$ is the input rf power, $Q_{E}$ is the external quality factor, $\epsilon_{0}$ is the vacuum permittivity, and $i=\sqrt{-1}$. Here we assumed $Q_{E}$ is constant in time and that $Q_{0} \gg 1$. The variation of input phase is contained in the complex function $P_{\text {in }}(t)$, so $\Omega$ is 

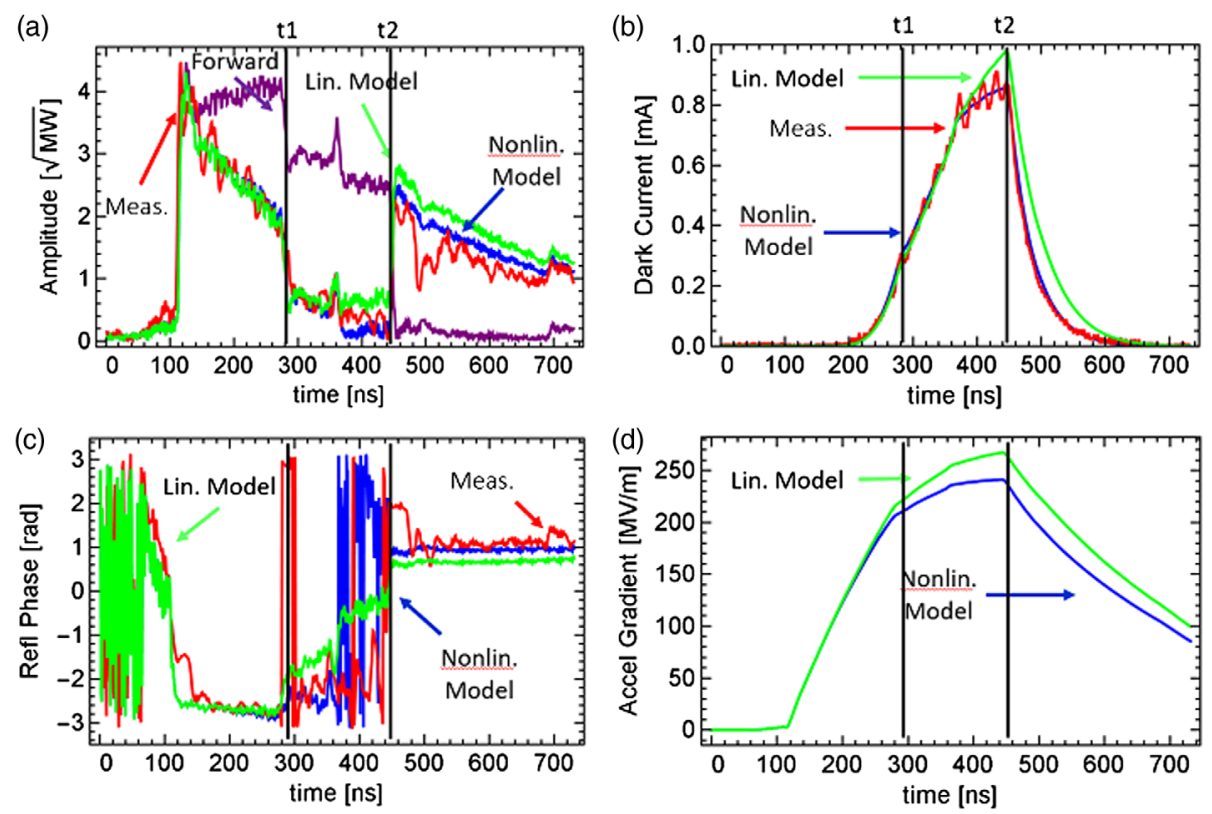

FIG. 6. Measured and reconstructed rf and current monitor signals for an example rf pulse. The measured current will be a small percentage of the field emitted current inside the cavity, which we estimated to be on the order of .1-1\% [70]. Measured signals are in purple and red. Results of the nonlinear model are in blue and results from a linear model are in green. $t_{1}$ and $t_{2}$ are defined as the section where the input rf power is decreased to a lower power level and the gradient is flat, which for this pulse is $150 \mathrm{~ns}$ long.

also constant for this calculation. The behaviors of $Q_{0}(t)$ and $\omega_{0}(t)$ are described in detail in [62].

This equation is derived directly from Maxwell's equations for electromagnetic fields in a resonant cavity. In the case that the quality factor and resonant frequency are constant, this equation can be reduced to the standard linear equivalent circuit [71]:

$$
\left[\frac{d^{2}}{d t^{2}}+\frac{\omega_{0}}{Q_{L}} \frac{d}{d t}+\omega_{0}^{2}\right] \tilde{E}(t)=-\sqrt{\frac{8 P_{\mathrm{in}} \omega_{0}^{3}}{\mu_{0} Q_{E}}} e^{-i \Omega t} .
$$

Using the nonlinear model, we were able to successfully reconstruct the accelerating gradient, proportional to $\tilde{E}(t)$, using the measured rf and dark current signals. We present an example of the accelerating gradient reconstruction in Fig. 6. In this figure, the difference between the linear and nonlinear models are apparent in the reconstructed dark current signal.

The model does not include any assumptions to what physical phenomenon causes the frequency shift presented in the nonlinear model. Our hypothesis is that the presence of strong currents inside the resonant cavity will not only absorb rf power but will also drive the fields in the cavity. This beam driven power may manifest as a frequency shift in the measured signals. Study of this phenomenon is outside the scope of this paper, but in the future, to test this hypothesis, we will perform self-consistent particle in cell simulations of dark currents in the rf structure similar to [31].

\section{RESULTS}

\section{A. Low power measurements}

We performed two sets of low power measurements, one before and one after the high power test. In the initial low power set, we measured the resonant frequencies of the three modes for Cryo-Cu-SLAC-\#2, and performed a bead pull measurement at room temperature. Results of these tests are included in Fig. 7. The procedure for bead pull measurements is outlined in [72]. In the second set of tests, we used the VNA to measure the $Q_{0}$ of all three modes from 10 to $250 \mathrm{~K}$. The results are shown in Fig. 8.

\section{B. High power measurements}

Figure 9 shows the processing history of $\mathrm{Cryo}-\mathrm{Cu}-$ SLAC-\#2, including the temperature, number of accumulated rf breakdowns, and the accelerating gradient in time. In Fig. 9(b) the nonlinear model was used to calculate the accelerating gradient after $70 \times 10^{6}$ pulses. For pulses before that the linear model was used and may overestimate the gradient. Figure 10 shows a zoom in after $70 \times 10^{6}$ pulses. We used data from this time period to calculate the rf breakdown rate. Breakdown rate was measured for periods of 1-3 million pulses where the gradient and rate of breakdowns were relatively constant. We measured both trigger breakdown rates and total breakdown rates as discussed in [63-65].

The measured value for the trigger breakdown rate is $2 \times 10^{-4} / \mathrm{pulse} / \mathrm{m}$ and total breakdown rate of 

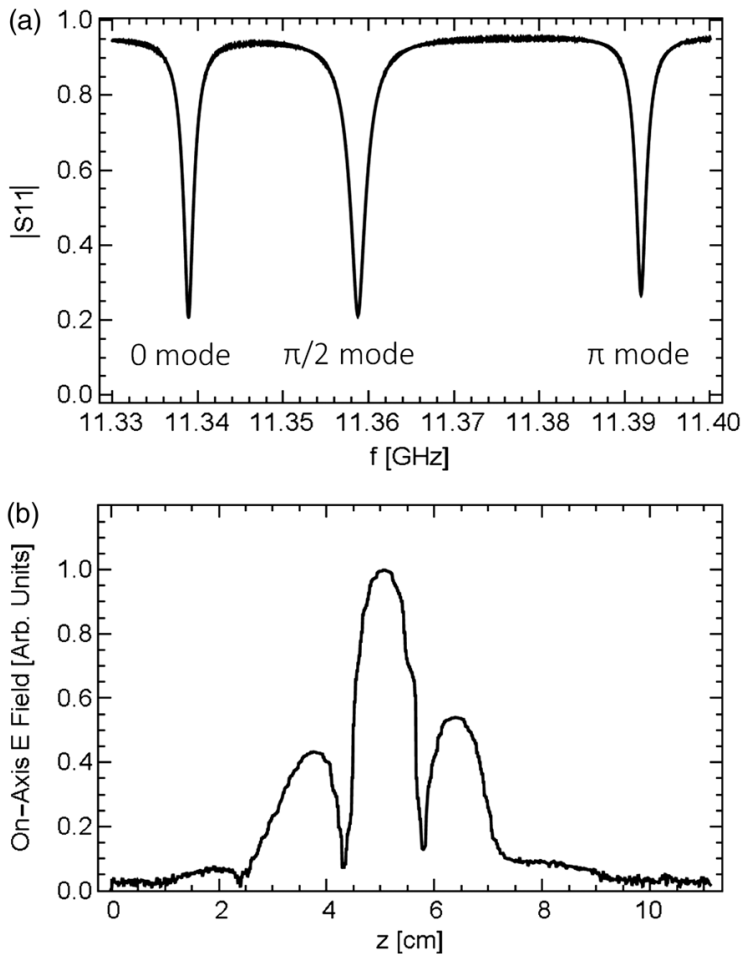

FIG. 7. (a): Measured $|S 11|$ vs frequency and (b): measured onaxis electric fields for Cryo-Cu-SLAC-\#2 both at room temperature.

$10^{-3} / \mathrm{pulse} / \mathrm{m}$ both for $250 \mathrm{MV} / \mathrm{m}$ and a shaped pulse with $150 \mathrm{~ns}$ flat gradient. $250 \mathrm{MV} / \mathrm{m}$ accelerating gradient corresponds to a $507 \mathrm{MV} / \mathrm{m}$ peak surface electric field for this accelerating structure. Figure 11 compares these rf breakdown rates to previous measurements in room temperature copper, hard copper, and hard $\mathrm{CuAg}$ accelerating structures of the same shape $(2.75 \mathrm{~mm}$ aperture radius) [36]. The data shows that for a given breakdown probability near or below $10^{-4} / \mathrm{m} /$ pulse the sustained accelerating gradient increases going from soft $\mathrm{Cu}$ to hard $\mathrm{Cu}$ and to hard $\mathrm{CuAg}$ structures. Finally, the

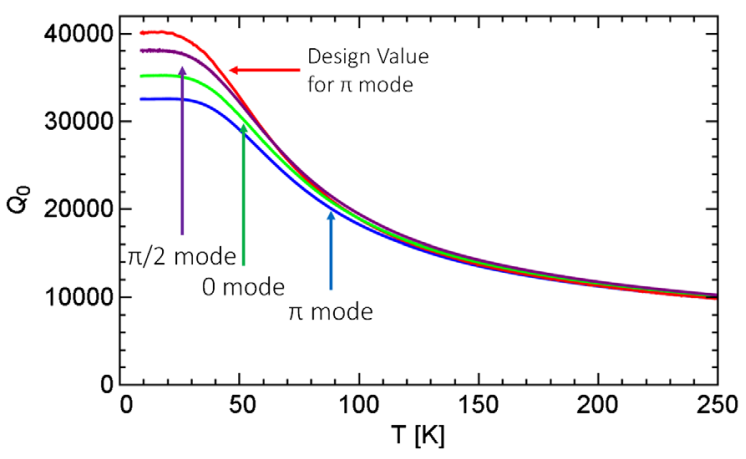

FIG. 8. Measured $Q_{0}$ versus temperature for the three modes of Cryo-Cu-SLAC-\#2, and the design value for the $\pi$ mode.
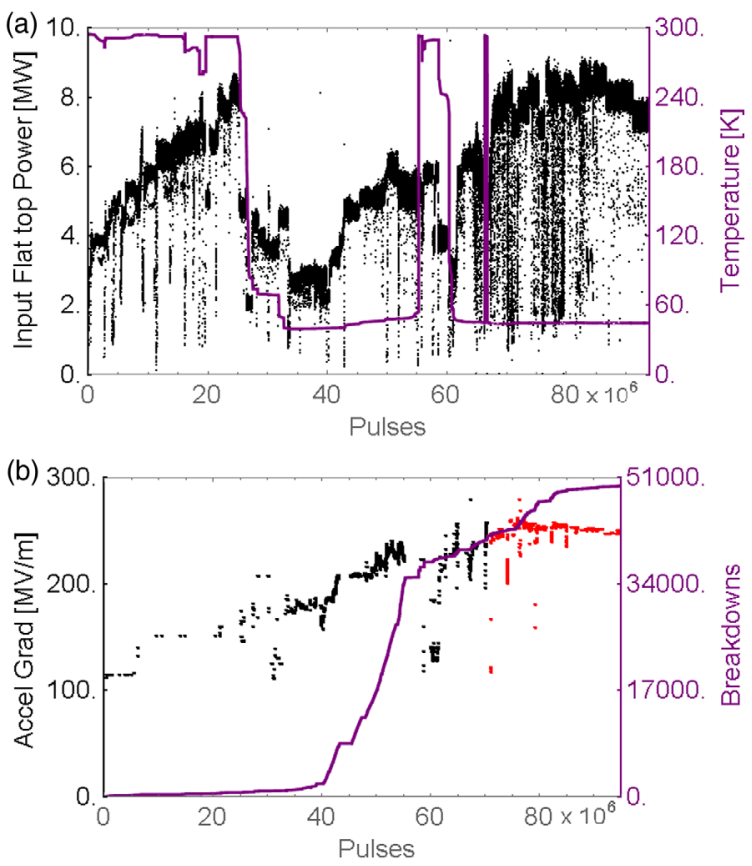

FIG. 9. The processing history for Cryo-Cu-SLAC-\#2. (a): Black is the input flat steady-state power (average between $t_{1}$ and $t_{2}$ as defined in Fig. 6) and purple is the temperature. (b): Purple is the number of accumulated breakdowns. Black and red points are the calculated average gradient (average between $t_{1}$ to $t_{2}$ ). Red indicates the time period where BDR was measued and a zoom-in is shown in Fig. 10.

copper structure at cryogenic temperatures reaches larger accelerating gradients for the same breakdown rate, and is the highest recorded gradient in $X$-band rf structures for this breakdown rate. This behavior is different than that found in [59], where temperature did not have an effect on the rf breakdown rate.

Due to conditions of this experiment, it is very difficult to obtain enough data to fully characterize the statistical behavior of the phenomenon. A zoom in on the graph will give the impression that there is a fine structure in the data, which is not present.

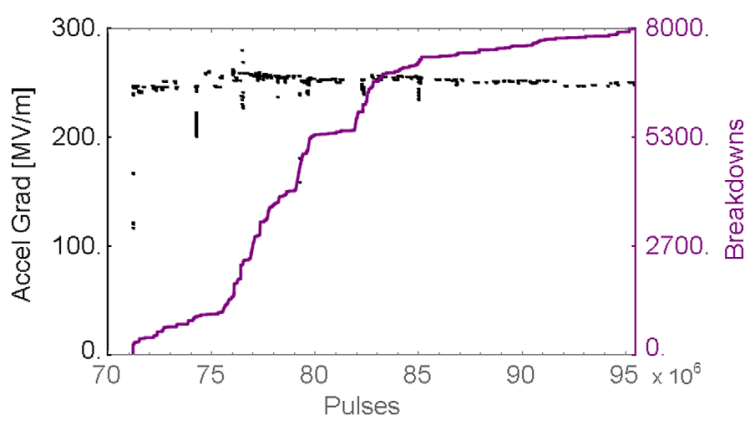

FIG. 10. Purple is the accumulated breakdowns and black the calculated average accelerating gradient (average over $t_{1}$ to $t_{2}$ ) from the nonlinear model. This is a zoom in of Fig. 9(b) for the time period where the BDR was measured. 

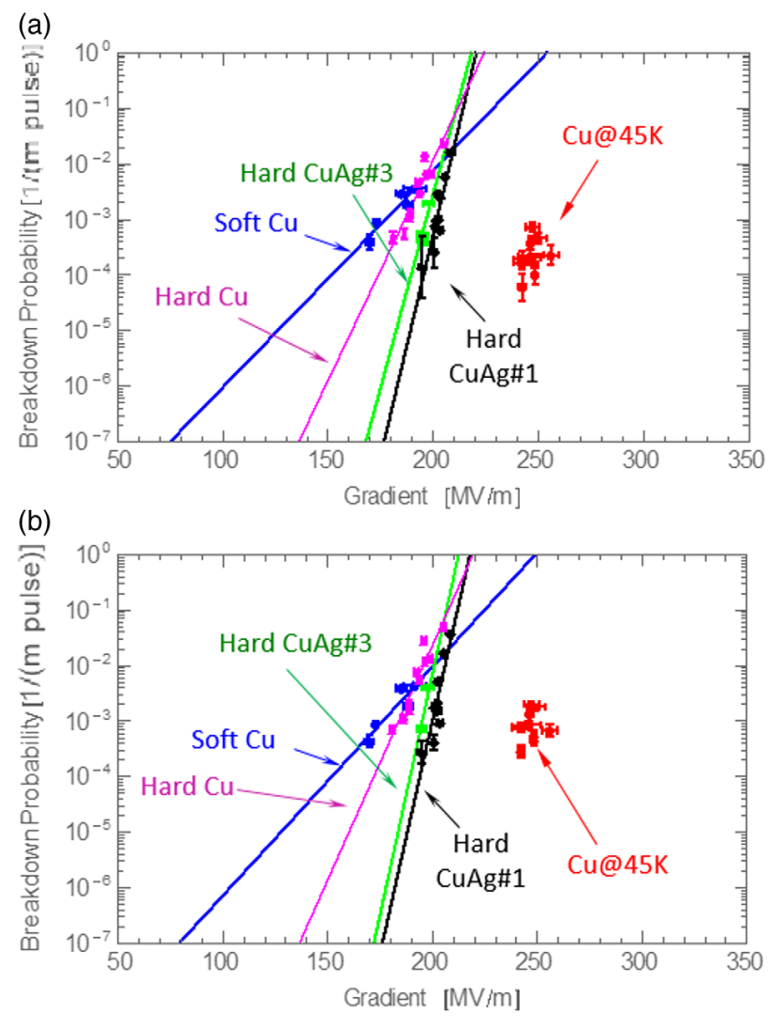

FIG. 11. Breakdown rate vs gradient:(a): first, trigger rf breakdowns; (b): all rf breakdowns. For the breakdown probability $\sim 10^{-4} /$ pulse/m cryogenic structure clearly outperforms record data from hard $\mathrm{CuAg}$ [36].

\section{DISCUSSION}

\section{A. Effect of dynamically changed quality factor on calculated gradient}

This is the first of our experiments where the field emission current beam loading has become a significant factor. We developed a model to account for the resultant change in $Q_{0}$. We optimize input rf pulse by making dark current flat in a second part of the pulse, between $\mathrm{t} 1$ and $\mathrm{t} 2$ on Fig. 6. At high gradient the $Q_{0}$ is decreased by loading from field emission currents, and the pulse shape is optimized for this decreased $Q_{0}$. If for the same pulse shape, we use the unchanged $Q_{0}$, the calculated gradient would not increase significantly. In the example pulse shown in Fig. $6, Q_{0}$ decreases from 30,300 to 17,900 , but the average gradient (average over $t_{1}$ to $t_{2}$ ) of the resonant cavity only changes from $248 \mathrm{MV} / \mathrm{m}$ to $237 \mathrm{MV} / \mathrm{m}$ when comparing the constant $Q_{0}$ linear and time-dependent $Q_{0}$ nonlinear models. The change in gradient is relatively small for two reasons: the external quality factor $Q_{E}=15347$ is smaller $Q_{0}$ at this temperature and the decrease in $Q_{0}$ changes the coupling closer to critical $\beta=1$. For the same power level, we would only expect the asymptotic accelerating gradient to increase by about $20 \%$ for this drop in $Q_{0}$. Second, the forward $\mathrm{rf}$ pulse is optimized to create a flat gradient for the smaller $Q_{0}$. For this same pulse, a cavity where the $Q_{0}$ does not decrease would not reach the asymptotic gradient. Thus the average over where the gradient should be flat is instead an average over a growing function of gradient, and is lower than the asymptotic gradient.

\section{B. Structure damage}

The low power measurement of $Q_{0}$ at cryogenic temperatures, shown in Fig. 8, occurred after Cryo-Cu-SLAC-\#2 had been exposed to large accelerating gradients. The design value of $Q_{0}$ at $45 \mathrm{~K}$, obtained from our previous low power cryogenic copper experiments, is near 36,000 , but the measured value is only 30,263 . We conjecture that the smaller $Q_{0}$ was caused either by damage during high power operation, or by surface degradation when the tested cavity was vented to perform low power measurements. This conjecture is further supported by the lower $Q_{0}$ in the $\pi$ mode when compared to the other resonant modes. The $\pi$ mode would be the most affected by damage or surface degradation to the middle cell, where the $\pi / 2$ mode would be affected the least, which we see in Fig. 8 .

We performed an autopsy on the structure. The preliminary results are reported in [73]. We did not find any unusual concentrations of breakdown damage. The breakdown pits are mostly located in area of high electric and high peak Poynting vector. This pattern is typical for structures of the same geometry [74]. What was unusual for this particular structure, is an absence of damage due to pulse heating in areas of maximum rf magnetic field, typically observed at similar gradients and rf pulse lengths [74]. We speculate that the absence of the damage is related to the properties of the copper at cryogenic temperatures. We also speculate that the lower $Q_{0}$ mentioned in the previous paragraph stems from surface degradation from exposure to air, rather than damage from high power operation.

\section{Future directions}

Next, we plan to confirm reproducibility of the high power results with a third identical structure, Cryo- $\mathrm{Cu}-$ SLAC-\#3, that has been manufactured and the geometry was successfully verified via a bead pull test. The current experiment was limited to running in day-long increments and thus we were not able to accurately measure BDRs less than $\sim 10^{-5} / \mathrm{pulse} / \mathrm{m}$, which corresponds to a few breakdowns per day, due to a lack of statistics. The next installation will be operated continuously for many days and will be able to measure the BDR for lower gradients.

This continuous operation will allow us to measure the exponent, $\zeta$ for the power law dependence BDR $\propto G^{\zeta}$ and repeat this measurement at different operating temperatures from $25 \mathrm{~K}$ to $77 \mathrm{~K}$. This enables us to verify the theory proposed in [43], which predicts a stronger power law dependence between BDR and accelerating gradient for both harder and colder structures. 
We plan to continue experiments at cryogenic temperatures with structures of different materials, such as $\mathrm{CuAg}$, and at a lower frequency, S-band. The details of the S-band experiment are presented in [75].

\section{CONCLUSION}

We studied the physics of vacuum rf breakdown using 11.4 GHz cryogenic normal conducting accelerating cavities. Decreasing the temperature of copper causes an increase in hardness and thermal conductivity, while decreasing the coefficient of thermal expansion and $\mathrm{rf}$ surface resistance. These changes of the material properties decrease both thermally induced stress and crystal defect mobility, which may lead to the decreased rf breakdown rates. The structure was processed to $250 \mathrm{MV} / \mathrm{m}$ and corresponding peak surface electric fields of $500 \mathrm{MV} / \mathrm{m}$ with $10^{8} \mathrm{rf}$ pulses and had a trigger breakdown rate of $2 \times 10^{-4} /$ pulse $/ \mathrm{m}$ for a shaped pulse with $150 \mathrm{~ns}$ flat gradient. For the same breakdown rate, the accelerating gradient is larger than that of room temperature structures of the same geometry. These results are different than those presented in [59], where decreased temperature of the accelerating structure did not affect the rf breakdown rate. The better performance observed in the cryogenic accelerating structure supports the hypothesis that rf breakdowns are caused by movement of crystal defects.

A factor of two increase in accelerating gradient over the current copper accelerators of the same frequency will enable a multitude of applications. One compelling application is, if the peak surface electric field of an rf photoinjector for LCLS was increased by a factor of two, the required undulator length could be reduced by more than a factor of two and increase the X-ray photon energy [20].

\section{ACKNOWLEDGMENTS}

This work was supported by the U.S. NSF Grant No. PHY-1549132, the Center for Bright Beams and DOE/SU Contract No. DE-AC02-76-SF00515.

[1] P. Emma et al., First lasing and operation of an ångstromwavelength free-electron laser, Nat. Photonics 4, 641 (2010).

[2] D. H. Whittum, in 22nd International Conference on Infrared and Millimeter Waves.

[3] V. A. Dolgashev and S. G. Tantawi, in Proceedings of the 8th European Particle Accelerator Conference, Paris, 2002 (EPS-IGA and CERN, Geneva, 2002), p. 2139.

[4] J. W. Wang, High Energy Phys. Nucl. Phys. 30, 11 (2006).

[5] F. Wang, C. Adolphsen, and C. Nantista, Performance limiting effects in $X$-band accelerators, Phys. Rev. ST Accel. Beams 14, 010401 (2011).

[6] The Stanford Two Mile Accelerator, edited by R. B. Neal, D. W. Dupen, H. A. Hogg, and G. A. Loew (W.A.
Benjamin, Inc., New York, 1968); Report No. SLACREPRINT-1968-001.

[7] S. Doebert et al., in Proceedings of the 21st Particle Accelerator Conference, Knoxville, TN, 2005 (IEEE, Piscataway, 2005), p. 372.

[8] T. C. S. T. Guignard, The clic study team, CERN Report No. CERN 2000-008, 2000.

[9] F. V. Hartemann and F. Albert, LLNL Technical Report No. LLNL-TR-416320.

[10] G. D'Auria, X-band technology applications at FERMI@Elettra FEL project, Nucl. Instrum. Methods Phys. Res., Sect. A 657, 150 (2011).

[11] J. P M Beijers, S. Brandenburg, K. Eikema, S. Hoekstra, K. Jungmann, T. Schlathlter, R. G E Timmermans, L. Willmann, R. Hoekstra, P. H M V Loosdrecht, B. Noheda, and T. Palstra, in Proceedings of the 32nd Free Electron Laser Conference, Malmö, Sweden (Max-lab, Sweden, 2010), p. 163.

[12] U. Amaldi, S. Braccini, A. Citterio, K. Crandall, M. Crescenti, M. Dominietto, A. Ciuliacci, F. Magrin, C. Mellace, P. Pearce, G. Pitta, E. Rosso, M. Weiss, and R. Zennaro, Cyclinacs: Fast-cycling accelerators for hadrontherapy, arXiv:0902.3533.

[13] A. E. Vlieks, C. Adolphsen, V. Dolgashev, J. Lewandowski, C. Limborg, and S. Weathersby, in Proceedings of the 3rd International Particle Accelerator Conference, New Orleans, LA, 2012 (IEEE, Piscataway, 2012).

[14] P. McIntosh, R. Akre, W. Brooks, P. Emma, and C. Rago, Report No. SLAC-PUB-11270; in Proceedings of the 21st Particle Accelerator Conference, Knoxville, TN, 2005 (IEEE, Piscataway, 2005).

[15] V. A. Dolgashev, in ICFA Beam Dynamics Mini-Workshop on Deflecting/Crabbing Cavity Applications in Accelerators Cockcroft Institute, 2010, https://indico.cern.ch/ event/83532/.

[16] V. A. Dolgashev, G. Bowden, Y. Ding, P. Emma, P. Krejcik, J. Lewandowski, C. Limborg, M. Litos, J. Wang, and D. Xiang, Design and application of multimegawatt $X$-band deflectors for femtosecond electron beam diagnostics, Phys. Rev. ST Accel. Beams 17, 102801 (2014).

[17] P. Craievich, M. Petronio, S. G. Biedron, D. Castronovo, M. D. Forno, S. D. Mitri, N. Faure, D. L. Civita, G. Penco, L. Rumiz, L. Sturari, R. Vescovo, and D. Wang, Implementation of radio-frequency deflecting devices for comprehensive high-energy electron beam diagnosis, IEEE Trans. Nucl. Sci. 62, 210 (2015).

[18] M. Shumail, G. Bowden, C. Chang, J. Neilson, S. Tantawi, and C. Pellegrini, in Proceedings of the 2nd International Particle Accelerator Conference, San Sebastián, Spain (EPS-AG, Spain, 2011).

[19] S. Tantawi, M. Shumail, J. Neilson, G. Bowden, C. Chang, E. Hemsing, and M. Dunning, Experimental Demonstration of a Tunable Microwave Undulator, Phys. Rev. Lett. 112, 164802 (2014).

[20] J. B. Rosenzweig, A. Cahill, V. Dolgashev, C. Emma, A. Fukasawa, R. Li, C. Limborg, J. Maxson, P. Musumeci, A. Nause, R. Pakter, R. Pompili, R. Roussel, B. Spataro, and $\mathrm{S}$. Tantawi, Next generation high brightness electron beams from ultra-high field cryogenic radiofrequency photocathode sources, arXiv:1603.01657. 
[21] G. A. Loew and J. W. Wang, http://inspirehep.net/record/ 454313/.

[22] V. Balakin, I. Koop, A. Novokhatski, A. S. Skrinski, and V. Smirnov, http://inspirehep.net/record/132627/.

[23] C. Adolphsen, in Proceedings of the 20th Particle Accelerator Conference, PAC-2003, Portland, OR, 2003 (IEEE, New York, 2003), p. 668.

[24] V. A. Dolgashev, AIP Conf. Proc. 1507, 76 (2012).

[25] J. Wang, J. Lewandowski, J. V. Pelt, C. Yoneda, G. Riddone, D. Gudkoc, T. Higo, and T. Takatomi, in Proceedings of the International Particle Accelerator Conference, Kyoto, Japan (ICR, Kyoto, 2010).

[26] T. Higo, Y. Higashi, S. Matsumoto, K. Yokoyama, S. Doebert, A. Grudiec, G. Riddone, W. Wuensch, T. Zennaro, C. Adolphsen, V. Dolgashev, A. Jensen, L. Laurent, S. Tantawi, F. Wang, and J. Wang, in Proceedings of the International Particle Accelerator Conference, Kyoto, Japan (ICR, Kyoto, 2010), p. 3702-3704.

[27] T. Higo, in Proceedings of the 25th International Linear Accelerator Conference, LINAC-2010, Tsukuba, Japan (KEK, Tsukuba, 2010).

[28] N. Catalan, in International Workshop on Breakdown Science and High Gradient Accelerator Technology (HG2016), https://indico.fnal.gov/event/ANLHEP963/ timetable/\#all.detailed.

[29] W. Wuensch, in International Workshop on Breakdown Science and High Gradient Technology in Tsukuba, Japan, https://indico.cern.ch/event/165513/timetable/\#all.detailed.

[30] V. Dolgashev, S. Tantawi, Y. Higashi, and B. Spataro, Geometric dependence of radio-frequency breakdown in normal conducting accelerating structures, Appl. Phys. Lett. 97 (2010).

[31] V. A. Dolgashev and S. G. Tantawi, in Proceedings of the 19th Particle Accelerator Conference, Chicago, IL, 2001 (IEEE, Piscataway, 2001), p. 3807.

[32] V. Dolgashev and S. Tantawi, Effect of rf parameters on breakdown limits in high-vacuum $X$-band structures, AIP Conf. Proc. 691, 151 (2003).

[33] V. A. Dolgashev, in Proceedings of the 2003 Particle Accelerator Conference, Portland, OR (IEEE, New York, 2003), p. 1267-1269.

[34] V. A. Dolgashev and S. G. Tantawi, in Proceedings of the 8th European Particle Accelerator Conference, Paris, 2002 (EPS-IGA and CERN, Geneva, 2002), p. 2139.

[35] A. Grudiev, S. Calatroni, and W. Wuensch, New local field quantity describing the high gradient limit of accelerating structures, Phys. Rev. ST Accel. Beams 12, 102001 (2009).

[36] V. Dolgashev, 2nd European Advanced Accelerator Concepts Workshop (EAAC 2015), 2015, https://agenda .infn.it/conferenceOtherViews.py? confId=8146\&view $=$ standardshort.

[37] C. Adolphsen, W. Baumgartner, K. Jobe, F. L. Pimpec, R. Loewen, D. McCormick, M. Ross, T. Smith, J. W. Wang, and T. Higo, in Proceedings of the 19th Particle Accelerator Conference, Chicago, IL, 2001 (IEEE, Piscataway, 2001), p. 478.

[38] W. Wuensch, A. Degiovanni, S. Calatroni, A. Korsbäck, F. Djurabekova, R. Rajamäki, and J. Giner-Navarro, Statistics of vacuum breakdown in the high-gradient and low-rate regime, Phys. Rev. Accel. Beams 20, 011007 (2017).
[39] A. Degiovanni, W. Wuensch, and J. Giner Navarro, Comparison of the conditioning of high gradient accelerating structures, Phys. Rev. Accel. Beams 19, 032001 (2016).

[40] V. Dolgashev, S. Tantawi, Y. Higashi, and B. Spataro, Geometric dependence of radio-frequency breakdown in normal conducting accelerating structures, Appl. Phys. Lett. 97, 171501 (2010).

[41] L. Laurent, S. Tantawi, V. Dolgashev, C. Nantista, Y. Higashi, M. Aicheler, S. Heikkinen, and W. Wuensch, Experimental study of rf pulsed heating, Phys. Rev. ST Accel. Beams 14 (2011).

[42] S. Heikkinen, Study of high power rf induced thermal fatigue in the high gradient accelerating structures, Ph.D. thesis, Helsinki University of Technology, Finland, 2008.

[43] K. Nordlund and F. Djurabekova, Defect model for the dependence of breakdown rate on external electric fields, Phys. Rev. ST Accel. Beams 15 (2012).

[44] E. Engelberg and Y. Ashkenazy, in 6th International Workshop on Mechanisms of Vacuum Arcs (2017), https://indico .cern.ch/event/521667/contributions/2409278/attachments/ 1433908/2203931/eli_mevarc.pdf.

[45] R. A. Matula, Electrical resistivity of copper, gold, palladium, and silver, J. Phys. Chem. Ref. Data 8, 1147 (1979).

[46] R. P. Reed and R. P. Mikesell, Low Temperature Mechanical Properties of Copper and Selected Copper Alloys (National Bureau of Standards, Boulder, 1967), Vol. NBS Monograph 101.

[47] D. Pritzkau, RF pulsed heating, Ph.D. thesis, Stanford University, 2001.

[48] G. Reuter and E. Sondheimer, The theory of the anomalous skin effect in metals, Proc. R. Soc. A 195, 336 (1948).

[49] B. Pippard, The surface impedance of superconductors and normal metals at high frequencies II. The anomalous skin effect in normal metals, Proc. R. Soc. A 191, 385 (1947).

[50] T. Tanaka, T. Sakai, K. Nogami, K. Hayakawa, Y. Hayakawa, K. Nakao, K. Takatsuka, M. Fukuda, F. Satoh, T. Takatomi, N. Terunuma, J. Urakawa, and M. Yoshida, in Proceedings of International Particle Accelerator Conference (IPAC'17), Copenhagen, Denmark, 2017 (JACoW, Geneva, Switzerland, 2017), p. 518, ISBN: 978-395450-182-3.

[51] A. Iino, S. Yamaguchi, T. Shintomi, T. Higo, Y. Funahashi, S. Matsumoto, S. Michizono, H. Endo, and K. Ueno, in Proceedings of 13th Annual Meeting of Particle Accelerator Society of Japan, p. 863, https://inis.iaea.org/search/ search.aspx?orig_q=RN:48076340.

[52] F. Biquard and A. Septier, Amelioration de la conductivite superficielle du cuivre et de l'aluminium en hyperfrequences, par abaissement de temperature, Nucl. Instrum. Methods 44, 18 (1966).

[53] J. Benard, N. H. E. Minyawi, and N. T. Viet, Reduction of $\mathrm{RF}$ losses at $35 \mathrm{GHz}$ in high purity copper resonant cavities by cooling to cryogenic temperature, Rev. Phys. Appl. 13, 483 (1978).

[54] J. Guo, S. Tantawi, D. Martin, and C. Yoneda, Cryogenic RF material testing with a high-Q copper cavity, AIP Conf. Proc. 1299, 330 (2010). 
[55] A. J. Saversky and I. S. Shchedrin, in Proceedings of the 15th Particle Accelerator Conference, PAC-1993, Washington, DC, 1993 (IEEE, New York, 1993), p. 1030.

[56] A. Cahill, A. Fukasawa, J. Rosenzweig, G. Bowden, V. Dolgashev, M. Franzi, S. Tantawi, P. Welander, C. Yoneda, J. Guo, and Y. Higashi, in Proceedings of International Particle Accelerator Conference (IPAC'16), Busan, Korea, 2016 (JACoW, Geneva, Switzerland, 2016), pp. 487-490, ISBN 978-3-95450-147-2.

[57] A. H. McEuen, P. Lui, E. Tanabe, and V. Vaguine, Highpower operation of accelerator structures at liquid nitrogen temperature, IEEE Trans. Nucl. Sci. 32, 2972 (1985).

[58] R. A. Kishek, Y. Y. Lau, L. K. Ang, A. Valfells, and R. M. Gilgenbach, Multipactor discharge on metals and dielectrics: Historical review and recent theories, Phys. Plasmas 5, 2120 (1998).

[59] H. H. Braun, S. Döbert, I. Wilson, and W. Wuensch, Frequency and Temperature Dependence of Electrical Breakdown at 21, 30, and $39 \mathrm{GHz}$, Phys. Rev. Lett. 90, 224801 (2003).

[60] V. A. Dolgashev, A. D. Cahill, S. Weathersby, J. Lewandowski, A. Haase, C. Yoneda, and S. Tantawi, International Workshop on Breakdown Science and High Gradient Technology (HG2015), Tsinghua University, Beijing, China, 2015 (SLAC National Accelerator Laboratory, 2015).

[61] A. Cahill, V. Dolgashev, J. Rosenzweig, S. Tantawi, and S. Weathersby, in Proc. of International Particle Accelerator Conference (IPAC'17), Copenhagen, Denmark, 2017 (JACoW, Geneva, Switzerland, 2017), p. 4395, ISBN: 978-395450-182-3.

[62] A. D. Cahill, J. B. Rosenzweig, V. A. Dolgashev, Z. Li, S. G. Tantawi, and S. Weathersby, rf losses in a high gradient cryogenic copper cavity, Phys. Rev. Accel. Beams, 21, 061301 (2018).

[63] B. J. Munroe, A. M. Cook, M. A. Shapiro, R. J. Temkin, V. A. Dolgashev, L. L. Laurent, J. R. Lewandowski, A. D. Yeremian, S. G. Tantawi, and R. A. Marsh, High power breakdown testing of a photonic band-gap accelerator structure with elliptical rods, Phys. Rev. ST Accel. Beams 16, 012005 (2013).

[64] R. A. Marsh, M. A. Shapiro, R. J. Temkin, V. A. Dolgashev, L. L. Laurent, J. R. Lewandowski, A. D.
Yeremian, and S. G. Tantawi, $X$-band photonic band-gap accelerator structure breakdown experiment, Phys. Rev. ST Accel. Beams 14, 021301 (2011).

[65] V. Dolgashev, G. Gatti, Y. Higashi, O. Leonardi, J. Lewandowski, A. Marcelli, J. Rosenzweig, B. Spataro, S. Tantawi, and D. Yeremian, High power tests of an electroforming cavity operating at $11.424 \mathrm{GHz}$, J. Instrum. 11, P03010 (2016).

[66] V. A. Dolgashev, J. R. Lewandowski, D. W. Martin, S. G. Tantawi, S. P. Weathersby, and A. D. Yeremian, in Proceedings of the 3rd International Particle Accelerator Conference, New Orleans, LA, 2012 (IEEE, Piscataway, 2012), Vol. C1205201, p. 3368.

[67] C. Nantista, S. Tantawi, and V. Dolgashev, Low-field accelerator structure couplers and design techniques, Phys. Rev. ST Accel. Beams 7, 072001 (2004).

[68] D. Yeremian, Choke flange for high power rf components excited by TE01 mode, SLAC Technical Report, 2009.

[69] T. Wangler, RF Linear Accelerators (Wiley, New York, 1998).

[70] K. L. F. Bane, V. A. Dolgashev, T. Raubenheimer, G. V. Stupakov, and J. Wu, Dark currents and their effect on the primary beam in an X-band linac, Phys. Rev. ST Accel. Beams 8, 064401 (2005).

[71] A. D. Cahill, Ultra-high accelerating gradients in radiofrequency cryogenic copper structures, Ph.D. thesis, University of California, Los Angeles, 2017.

[72] C. W. Steele, A nonresonant perturbation theory, IEEE Trans. Microwave Theory Tech. 14, 70 (1966).

[73] V. A. Dolgashev, in 7th International Workshop on Mechanisms of Vacuum Arcs (2018), https://indico.cern.ch/event/ 680402/contributions/2976659/attachments/1657385/ 2653803/MevArc2018_Dolgashev_Single_Cell_Cryo_ Autopsy_final_22May2018.pdf.

[74] V. A. Dolgashev, cLIC 2016 Workshop (2016), https://indico .cern.ch/event/449801/contributions/1945274/attachments/ 1213013/1769903/Dolgashev_CLIC_2016_Recent_high_ Gradient_Test_at_SLAC_v0653_18jan2015.pdf.

[75] A. Cahill, G. Bowden, V. Dolgashev, A. Fukasawa, J. Rosenzweig, and S. Tantawi, in Proceeding of North American Particle Accelerator Conference (NAPAC'16), Chicago, IL, USA, 2016 (JACoW, Geneva, Switzerland, 2016), p. 991, ISBN: 978-3-95450-180-9. 